\title{
Postoperative atrial fibrillation: Is there a need for prevention?
}

\author{
David Amar, MD
}

\footnotetext{
From the Department of Anesthesiology and Critical Care Medicine, Memorial Sloan Kettering Cancer Center, New York, NY.

Disclosures: Dr Amar is on the scientific advisory board of breathing tube maker ETView Medical, Misgav, Israel. Author has nothing to disclose with reagrd to commercial support.

Received for publication Sept 8, 2015; accepted for publication Sept 9, 2015; available ahead of print Oct 15, 2015.

Address for reprints: David Amar, MD, Department of Anesthesiology and Critical Care Medicine, Memorial Sloan-Kettering Cancer Center, 1275 York Ave, M-304, New York, NY 10021 (E-mail: amard@mskcc.org). J Thorac Cardiovasc Surg 2016;151:913-5

$0022-5223 / \$ 36.00$

Copyright (C) 2016 by The American Association for Thoracic Surgery

http://dx.doi.org/10.1016/j.jtcvs.2015.09.041
}

Surgeons seemingly view postoperative atrial fibrillation (POAF) as a self-limited nuisance that frightens patients. Numerous articles are published yearly on the predictors and mechanisms of, and prevention strategies for, POAF but the incidence appears to have not changed significantly over several decades. This raises 2 questions: Are there effective ways to prevent POAF? If so, why are clinicians not employing them? I have worked in this area for the past 25 years and herein will include my thoughts on recent advances in our understanding of POAF and whether POAF should be prevented, as well as highlight key recommendations pertaining to the prevention and management of POAF largely adapted from the comprehensive guideline document written in 2014 by an American Association for Thoracic Surgery Taskforce.

\section{EPIDEMIOLOGY AND MECHANISMS}

POAF affects more than 1 million Americans annually and is associated with increased resource use and risk of early postoperative stroke. ${ }^{2}$ In acute settings, POAF is typically associated with 1 or more signs or symptoms such as palpitations, dizziness, shortness of breath, or hypotension and stroke if persistent. ${ }^{1,2}$ POAF has recently been shown to be associated with long-term risk of ischemic stroke, more so after noncardiac surgery than cardiac surgery. ${ }^{3}$ POAF occurs overall in 16\% (range, $8 \%-32 \%$ ) of patients following anatomic lung resection or esophagectomy (probably due to anatomic dissection of autonomic hilar nerve fibers of the deep and superficial cardiac plexi) and $<5 \%$ of patients who have nonanatomic lung resections, for example. ${ }^{1,2}$ It is likely for this reason that the application of minimally invasive techniques to thoracic and cardiac surgery have not been associated with reduced rates of POAF. ${ }^{4,5}$ Similar to atrial fibrillation (AF) unrelated to surgery, the most important predictor of POAF is older age. ${ }^{1,2,6}$ Although it may take years to develop AF in the general population, the intense stimuli of the perioperative period can precipitate POAF in a short time period in susceptible individuals. Older age is known to be associated with increasing atrial fibrosis and other

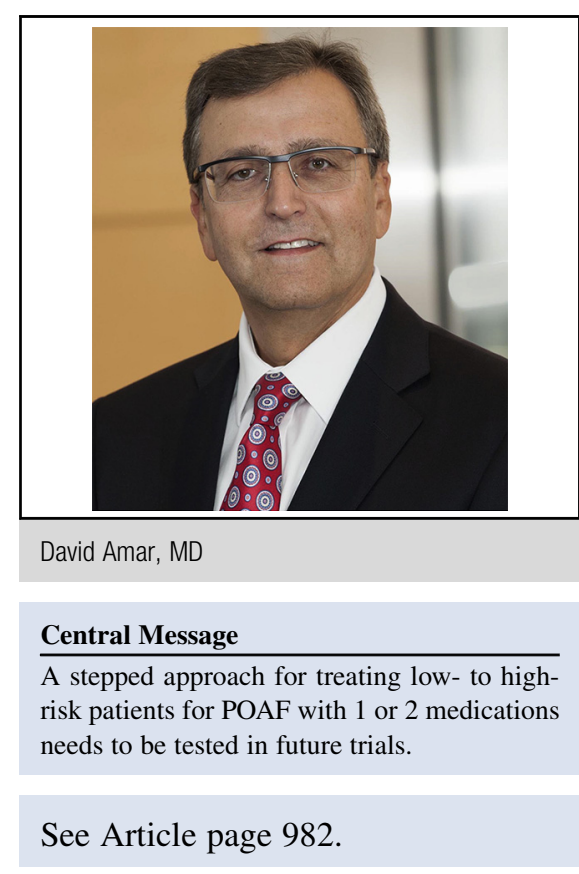

See Editorial Commentary page 990.

structural changes that largely provide the electrical substrate for arrhythmia generation in conjunction with other conditions such as atrial dilation, myocardial ischemia, or volume overload. ${ }^{1,2}$ Many studies have looked at biomarkers that may reflect the degree of atrial fibrosis to assess AF risk in surgical and nonsurgical patients. ${ }^{7}$ We and others have found that subclinical elevation in preoperative brain natriuretic peptide-even in the absence of other markers of cardiac dysfunction-confers a significant increased risk of POAF and we currently use this readily obtainable biomarker routinely at our institution as part of our risk stratification for prevention. ${ }^{8}$ Elevations in brain natriuretic peptide levels likely represent increases in left atrial size and diastolic dysfunction in patients largely without evidence of significant cardiac dysfunction. 9 The precipitating mechanisms in a susceptible patient during the postoperative period responsible for the initiation and maintenance of POAF are multifactorial and include adrenergic and vagal activation; cardiac inflammation; and more importantly, oxidative stress. ${ }^{1,2,10-14}$ Oxidative stress leads to high amounts of reactive oxygen species that can cause DNA damage, apoptosis, and myocyte dysfunction. ${ }^{7}$ These conditions lead to increased intracellular calcium levels and downregulation of the outward current that cause electrophysiologic remodeling and perpetuation of $\mathrm{AF}^{7,15}$ 


\section{PREVENTION METHODS}

Because the etiology for POAF is multifactorial and its mechanisms are not completely understood, it is unlikely that any single drug that affects a single pathway will provide effective POAF prevention across the full range of patients. For example, even with beta-blockade, the incidence of POAF remains high. It has been recommended that oral beta-blockers be continued after thoracic surgery to avoid withdrawal but not for the prevention of POAF (class I, level of evidence A). ${ }^{1}$ For intermediate- and high-risk patients undergoing lung resection, recommended prevention strategies include amiodarone (class IIa, level of evidence A) and diltiazem in patients who are not taking betablockers (class IIa, level of evidence B). ${ }^{1}$ The recommendation for amiodarone in patients undergoing esophagectomy was class IIB, level of evidence B due to the limited number of studies in these patients. Detailed dosage recommendations and precautions are provided in the 2014 Taskforce document $^{1}$ and should be consulted. Recommendations for amiodarone or diltiazem suggest that the drugs have a reasonable safety profile and are likely moderately effective but do not eliminate POAF. ${ }^{1,16,17}$

So why are physicians reluctant to employ prevention strategies at their institutions? It may be that they are trying to avoid use of antiarrhythmic agents because they carry a rare risk of being proarrhythmogenic (especially if electrolyte imbalance is present), bradycardic, or induce transient hypotension. Although amiodarone is commonly used after cardiac surgery, there is a rare $(<0.1 \%)$ risk of idiopathic pulmonary fibrosis even with a treatment period of $<1$ month (usually reversible with drug withdrawal). ${ }^{1,16}$ Probably out of concern that these complications could occur there has been increasing interest in nonantiarrhythmic medications shown to possess secondary properties of preventing AF after cardiothoracic surgery. ${ }^{18-22}$ In a recent double-blind study, ${ }^{19}$ we compared atorvastatin to placebo given 1 week before and 1 week after thoracic surgery and found a significant association with its use and the reduction of the composite end point of multiple complications, including POAF, pneumonia, acute respiratory distress syndrome, myocardial infarction, deep venous thrombosis, and in-hospital mortality. Our study lacked statistical power to show that atorvastatin specifically reduced POAF specifically (despite a $50 \%$ difference between the trial arms), but a beneficial effect was found by others in a larger study of patients undergoing cardiac surgery ${ }^{18}$ These positive clinical effects are likely due to weak to moderate antioxidant and/or anti-inflammatory activity at the myocardial tissue level. Atorvastatin received a class IIb, level of evidence $\mathrm{C}$ recommendation for POAF prevention in the The American Association for Thoracic Surgery Guidelines. ${ }^{1}$ More potent antioxidants such as $\mathrm{N}$-acetylcysteine when used alone or in combination with other medications have been shown to decrease serum levels of oxidative stress markers and reduced AF after cardiac surgery. ${ }^{20,21} \mathrm{~A}$ recent trial of antioxidant supplementation with oral n-3 polyunsaturated fatty acids started 1 week before on-pump cardiac surgery and then combined with vitamins $\mathrm{C}$ and $\mathrm{E}$ added 2 days before surgery showed that this regimen was associated with a significant reduction POAF as well as in plasma and atrial tissue markers of oxidative stress and inflammation. ${ }^{22}$

\section{STROKE PREVENTION}

An important milestone in the management of POAF has been the wider acceptance of evidence-based recommendations for antithrombotic therapy similar to those employed for AF unrelated to surgery. ${ }^{23}$ We found a $1.7 \%$ incidence of stroke related to POAF in a study of patients undergoing major lung resection. ${ }^{17}$ In a large, retrospective cohort study using a nonfederal administrative database of 1.7 million eligible patients who underwent cardiac and noncardiac surgery in California, 24,711 patients $(1.43 \%)$ developed new-onset perioperative $\mathrm{AF}$ and of these, $13,952(0.81 \%$; $95 \%$ confidence interval, $0.79 \%-0.82 \%)$ experienced an ischemic stroke, more commonly after noncardiac surgery. ${ }^{3}$ Because the potential for thromboembolism with new-onset AF develops early in the postoperative period (24-48 hours), prompt attempts to restore sinus rhythm within this period should be made. ${ }^{1,23}$ The 2014 The American Association for Thoracic Surgery Guidelines outline that if the arrhythmia persists beyond 24 to 48 hours, anticoagulant therapy should be considered if the patient's $\mathrm{CHA}_{2} \mathrm{DS}_{2}$-VASc score is 1 and highly recommended for a score $\geq 2$, after weighing the risk of postoperative bleeding. ${ }^{1}$

\section{PRACTICAL RECOMMENDATIONS AND FUTURE DIRECTIONS}

\section{Use of Prediction Rules}

Physicians are more inclined to use uncomplicated prediction algorithms ( $\leq 4$ risk factors) with high predictive accuracy that can be easily used in a busy clinical environment. The ability to accurately identify patients at high risk for POAF will allow clinicians to create an individualized approach that maximizes clinical benefits while reducing adverse drug reactions. The necessary nomograms and algorithms are yet to be developed.

\section{Use of Prevention Protocols}

Creating a multidisciplinary team to decide on and develop evidence-based POAF and stroke prevention strategies that work best at an individual institution will likely result in higher compliance. In comparison to oral prevention regimens, intravenous administration during the 
perioperative period is more likely to be employed by physicians and result in higher compliance among patients.

\section{Create a Clinical Trials Group for General Thoracic Surgery-Related POAF}

Akin to or in conjunction with The Cardiothoracic Surgery Network (www.ctsnet.org), this group would plan studies in various areas such as the epidemiology and genetic markers for POAF; validate existing prediction rules for POAF; promote the capture of consistent and accurate data on the clinical presentation, management, and adverse events related to POAF; and design and fund adequately powered multicenter prevention trials. For example, although a watchful waiting strategy may be acceptable in low-risk patients, a stepped approach for treating low-risk patient for POAF with 1 medication (perhaps a nonantiarrhythmic agent) and those patients with intermediate to high risk with 2 medications will likely lead to better prevention success. This question has not been formally studied in a large population of patients and should be prioritized.

The author thanks Dr Robert Downey for providing helpful comments.

\section{References}

1. Frendl G, Sodickson AC, Chung MK, Waldo AL, Gersh BJ, Tisdale JE, et al. 2014 AATS guidelines for the prevention and management of perioperative atrial fibrillation and flutter for thoracic surgical procedures. Executive summary. J Thorac Cardiovasc Surg. 2014;148:772-91.

2. Amar D. Perioperative atrial tachyarrhythmias. Anesthesiology. 2002;97: $1618-23$

3. Gialdini G, Nearing K, Bhave PD, Bonuccelli U, Iadecola C, Healey JS, et al. Perioperative atrial fibrillation and the long-term risk of ischemic stroke. JAMA. 2014;312:616.

4. Park BJ, Zhang H, Rusch VW, Amar D. Video-assisted thoracic surgery does not reduce the incidence of postoperative atrial fibrillation after pulmonary lobectomy. J Thorac Cardiovasc Surg. 2007;133:775-9.

5. Lamy A, Devereaux PJ, Prabhakaran D, Taggart DP, Hu S, Paolasso E, et al. Offpump or on-pump coronary-artery bypass grafting at 30 days. $N$ Engl J Med. 2012:366:1489-97.

6. Passman RS, Gingold DS, Amar D, Lloyd-Jones D, Bennett CL, Zhang H, et al. Prediction rule for atrial fibrillation after major noncardiac thoracic surgery. Ann Thorac Surg. 2005;79:1698-703.

7. Youn JY, Zhang J, Zhang Y, Chen H, Liu D, Ping P, et al. Oxidative stress in atrial fibrillation: an emerging role of NADPH oxidase. J Molec Cell Cardiol. 2013;62: $72-9$.
8. Amar D, Zhang H, Shi W, Downey RJ, Bains MS, Park BJ, et al. Brain natriuretic peptide and risk of atrial fibrillation after thoracic surgery. J Thorac Cardiovasc Surg. 2012;144:1249-53.

9. Raman T, Roistacher N, Liu J, Zhang H, Shi W, Thaler HT, et al. Preoperative left atrial dysfunction and risk of postoperative atrial fibrillation complicating thoracic surgery. J Thorac Cardiovasc Surg. 2012;143:482-7.

10. Amar D, Zhang H, Miodownik S, Kadish AH. Competing autonomic mechanisms precede the onset of postoperative atrial fibrillation. J Am Coll Cardiol. 2003;42:1262-8

11. Kim YM, Kattach H, Ratnatunga C, Pillai R, Channon KM, Casadei B. Association of atrial nicotinamide adenine dinucleotide phosphate oxidase activity with the development of atrial fibrillation after cardiac surgery. J Am Coll Cardiol. 2008;51:68-74.

12. Anselmi A, Possati G, Gaudino M. Postoperative inflammatory reaction and atrial fibrillation: simple correlation or causation? Ann Thorac Surg. 2009;88 326-33.

13. Anderson EJ, Efird JT, Davies SW, O'Neal WT, Darden TM, Thayne KA, et al. Monoamine oxidase is a major determinant of redox balance in human atrial myocardium and is associated with postoperative atrial fibrillation. J Am Heart Assoc. 2014;3:e000713.

14. Wu JH, Marchioli R, Silletta MG, Masson S, Sellke FW, Libby P, et al. Oxidative stress biomarkers and incidence of postoperative atrial fibrillation in the Omega3 Fatty Acids for Prevention of Postoperative Atrial Fibrillation (OPERA) trial. J Am Heart Assoc. 2015;4.

15. Yang KC, Dudley SC Jr. Oxidative stress and atrial fibrillation: finding a missing piece to the puzzle. Circulation. 2013;128:1724-6.

16. Berry MF, D'Amico TA, Onaitis MW. Use of amiodarone after major lung resection. Ann Thorac Surg. 2014:98:1199-206.

17. Amar D, Roistacher N, Rusch VW, Leung DH, Ginsburg I, Zhang H, et al. Effects of diltiazem prophylaxis on the incidence and clinical outcome of atrial arrhythmias after thoracic surgery. J Thorac Cardiovasc Surg. 2000;120:790-8.

18. Patti G, Chello M, Candura D, Pasceri V, D’Ambrosio A, Covino E, et al. Randomized trial of atorvastatin for reduction of postoperative atrial fibrillation in patients undergoing cardiac surgery: results of the ARMYDA-3 (Atorvastatin for Reduction of MYocardial Dysrhythmia After cardiac surgery) study. Circulation. 2006;114:1455-61.

19. Amar D, Park B, Zhang H, Shi W, Fleisher M, Thaler HT, et al. Beneficial effects of perioperative statins for major pulmonary resection. J Thorac Cardiovasc Surg. 2015;149:1532-8.

20. Ozaydin M, Icli A, Yucel H, Akcay S, Peker O, Erdogan D, et al. Metoprolol vs carvedilol or carvedilol plus $\mathrm{N}$-acetyl cysteine on post-operative atrial fibrillation: a randomized, double-blind, placebo-controlled study. Eur Heart J. 2013 34:597-604.

21. Liu XH, Xu CY, Fan GH. Efficacy of N-acetylcysteine in preventing atrial fibrillation after cardiac surgery: a meta-analysis of published randomized controlled trials. BMC Cardiovasc Disord. 2014;14:52.

22. Rodrigo R, Korantzopoulos $\mathrm{P}$, Cereceda M, Asenjo R, Zamorano J, Villalabeitia E, et al. A randomized controlled trial to prevent post-operative atrial fibrillation by antioxidant reinforcement. J Am Coll Cardiol. 2013;62: 1457-65.

23. January CT, Wann LS, Alpert JS, Calkins H, Cigarroa JE, Cleveland JC, et al 2014 AHA/ACC/HRS guideline for the management of patients with atrial fibrillation: a report of the American College of Cardiology/American Heart Association Task Force on Practice Guidelines and the Heart Rhythm Society. J Am Coll Cardiol. 2014;64:e1-76. 